\title{
Nuclear Structure Studies with Gamma-Ray Beams
}

\author{
Anton Tonchev ${ }^{1, \text { a }}$, Chitra Bhatia ${ }^{7}$, John Kelley ${ }^{5,4}$, Rajarshi Raut ${ }^{6}$, Gencho Rusev $^{2}$, Werner Tornow ${ }^{3,4}$, Nadia Tsoneva ${ }^{8,9}$ \\ ${ }^{1}$ Lawrence Livermore National Laboratory, Physics Division, Livermore, California 94550, USA \\ ${ }^{2}$ Los Alamos National Laboratory, Chemistry Division, Los Alamos, New Mexico 87545, USA \\ ${ }^{3}$ Duke University, Durham, North Carolina 27708, USA \\ ${ }^{4}$ Triangle Universities Nuclear Laboratory, Durham, North Carolina 27708, USA \\ ${ }^{5}$ North Carolina State University, Raleigh, North Carolina 27695, USA \\ ${ }^{6}$ UGC-DAE Consortium for Scientific Research, Kolkata Centre, Kolkata, India \\ ${ }^{7}$ McMaster University, Hamilton, ON, Canada \\ ${ }^{8}$ Institut für Theoretische Physik, Universität Giessen, Germany \\ ${ }^{9}$ Institute for Nuclear Research and Nuclear Energy, 1784 Sofia, Bulgaria
}

\begin{abstract}
In stable and weakly bound neutron-rich nuclei, a resonance-like concentration of dipole states has been observed for excitation energies below the neutron-separation energy. This clustering of strong dipole states has been named the Pygmy Dipole Resonance (PDR) in contrast to the Giant Dipole Resonance (GDR) that dominates the E1 response. Understanding the PDR is presently of great interest in nuclear structure and nuclear astrophysics. High-sensitivity studies of E1 and M1 transitions in closed-shell nuclei using monoenergetic and $100 \%$ linearly-polarized photon beams are presented.
\end{abstract}

\section{Introduction}

During the last years the Pigmy Dipole Resonance (PDR) has attracted a great deal of experimental and theoretical effort. This mode of excitation was first observed in neutron-capture reactions [1]. Nowadays this mode has been found as a non-statistical enhanced dipole strength below the neutron-separation energy for isotopes ranging from light nuclei $(8 \leq Z \leq 40)$ up to lead isotopes including the transitional nuclei [2-16]. A series of nuclear experiments have shown that this is a common mode in $N=82$ semi-magic ${ }^{136} \mathrm{Xe},{ }^{138} \mathrm{Ba},{ }^{140} \mathrm{Ce},{ }^{142} \mathrm{Nd}$ and ${ }^{144} \mathrm{Sm}$ nuclei at excitation energies between $5-8 \mathrm{MeV}[2,4,5,10]$. Similar conclusion was drawn for deformed nuclei where the effect of deformation on the PDR has been studied in the xenon isotopic chain [15]

There are different theoretical mechanisms explaining the amplitude and the distribution of the nuclear dipole strength of PDR observed in the experiments $[11,18,33]$. This new structure phenomenon is described as a neutron skin oscillation against a protonneutron saturated core. In the simple three-fluid nuclear model, three fluids were introduced to take into account the difference in the interaction of excess neutrons and the remainder of the neutrons and protons [25]. This simple hydrodynamic model was able to qualitatively explain the existence of two independent modes, one located at around $13 \mathrm{MeV}$ (GDR) and the other at about 4-5 MeV (PDR). A clear correlation of the total PDR strength and the thickness of the neutron skin is obtained in calculations for $N=82$ isotonic chains [2,4-6,32] and $\mathrm{Z}=50$ nuclei [18]. In addition, the recently observed fundamental differences [8] between the $\left(\gamma, \gamma^{\prime}\right)$ and $\left(\alpha, \alpha^{\prime}\right)$ data, provide a new challenge for model calculations. This comparison leads to the believe, that the PDR is split into two parts with different underlying structures.

The existence of PDR is now well established as $E 1$ excitations in spherical and transitional nuclei [19]. A concentration of the electric dipole excitations exhausting up to $1 \%$ of the isovector $E 1$ energy weighted sum rule [32]. A nonstatistical distribution of the $E 1$ strengths close to the threshold has a strong impact on the astrophysical $r$-process nucleosynthesis [26]. In addition, the PDR is sensitive to nuclear properties at the nuclear surface and at low density. Thus, its property may provide a useful constraint on the energy density functional, to identify the equation of state (EOS) of the nuclear and neutron matters [11].

In this paper, we focus on another scope of these nuclear structure studies with monoenergetic and polarized photon beams from the High-Intensity GammaRay Source (HIGS) facility: investigating the $E 1$ dipole strength distribution of the PDR overlapping with the spin-flip $M 1$ strength and E2 quadruple responses. The data are compared with predictions from statistical and quasi-phonon model [18].

\footnotetext{
a Corresponding author: tonchev2@1lnl.gov
} 


\section{Experimental technique}

The Nuclear Resonance Fluorescence method (NRF) is used at HIGS to study low-multipolarity ground-state transitions i.e., E1, M1 and to a lesser extent E2. The NRF technique represents an outstanding tool for measuring dipole transitions with a high detection sensitivity. The main advantage of this method is that both the excitation and the de-excitation processes proceed via the electromagnetic interaction, which is the most understood fundamental interaction in physics. The angular distribution of the scattered $\gamma$ rays from excited nuclei to the ground state or the secondary transitions from the first $2^{+}$states to the ground state were measured.

The monoenergetic and $100 \%$ linearly polarized photon beams were produced by intra-cavity Compton backscattering of intense free-electron-laser light from electron beams in the Duke storage ring. The energy of the backward scattered photons can be tuned within a wide range, presently from 1 to $100 \mathrm{MeV}$. The collimated photon flux on target exceeds $1000 \mathrm{eV}^{-1} \mathrm{~s}^{-1}$ within an energy spread from 1 to $4 \%$. The backscattered photons were collimated by a lead collimator of $30.5 \mathrm{~cm}$ in length with a cylindrical hole of $1.9 \mathrm{~cm}$ in diameter. After proper attenuation, the energy distribution of the photon beam was measured with a $123 \%$ HPGe detector placed in the beam. The photon flux was measured by Compton scattering of the beam from a 1.0-mm-thick copper plate, positioned 3 meters behind the scattering target. Photons scattered from the copper plate through $7.0^{\circ} \pm 1.6^{\circ}$ were detected with the same $123 \%$ HPGe detector. At energies above $8.1 \mathrm{MeV}$, the photon flux was also monitored by the ${ }^{197} \mathrm{Au}(\gamma, \mathrm{n})$ reaction.

\section{Electric dipole strength in $\mathrm{N}=82$ nuclei}

The ${ }^{138} \mathrm{Ba},{ }^{140} \mathrm{Ce},{ }^{142} \mathrm{Nd}$, and ${ }^{144} \mathrm{Sm}$ scattering targets were placed in an evacuated plastic tube at the center of an array of six large-volume HPGe detectors. These detectors were arranged around the target at three positions: $(\theta, \varphi)=\left(90^{\circ}, 90^{\circ}\right.$ and $\left.270^{\circ}\right),\left(90^{\circ}, 0^{\circ}\right.$ and $\left.180^{\circ}\right)$, and $\left(135^{\circ}, 0^{\circ}\right)$, where $\theta$ is the polar angle with respect to the horizontally-polarized incoming photon beam, and $\varphi$ is the azimuthal angle measured from the polarization vector. This detector configuration allows for the unambiguous determination of $E 1, M 1$, and $E 2$ transitions. The experimental results of the E1 strength distribution in $\mathrm{N}=82$ isotones is shown in Fig. 1. These results represent the fine structure of the E1 PDR distribution. If one integrates the E1 strengths between 4.0 $\mathrm{MeV}$ and the neutron separation energy for each of the $\mathrm{N}=82$ isotones, excluding the 2-phonon state, then the summed $\mathrm{B}(\mathrm{E} 1) \uparrow=960 \pm 153,600 \pm 119,576 \pm 98$, $536 \pm 96\left(10^{-3} \mathrm{e}^{2} \mathrm{fm}^{2}\right)$ for ${ }^{138} \mathrm{Ba},{ }^{140} \mathrm{Ce},{ }^{142} \mathrm{Nd}$, and ${ }^{144} \mathrm{Sm}$, respectively. One can see a decrease in strength with increasing proton number.

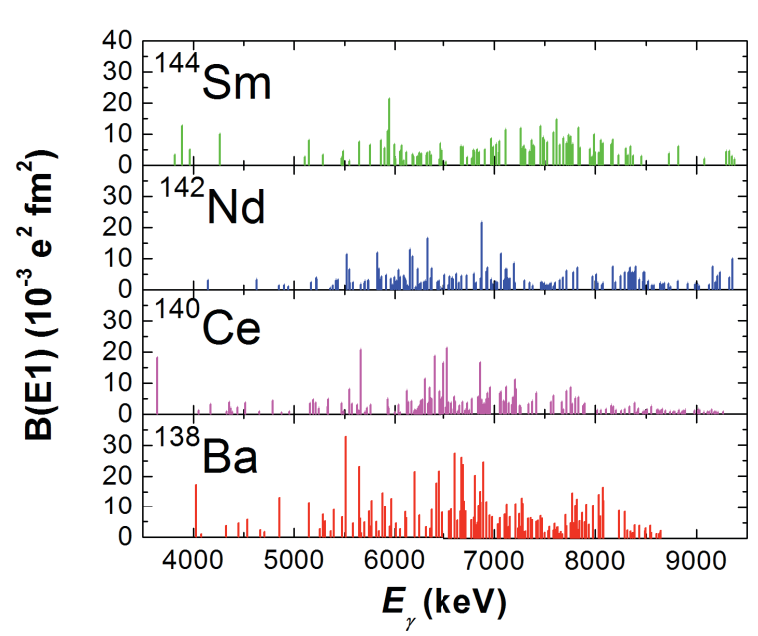

Figure 1. $B(E 1) \uparrow$ strength distribution in the $N=82$ isotones.

\section{Magnetic dipole strength below the neutron separation energy}

\subsection{Fine structure of the $M 1$ strength in ${ }^{90} \mathrm{Zr}$}

The distribution of the magnetic dipole transition strength in ${ }^{90} \mathrm{Zr}$ has been measured at excitation energies between 7 and $11 \mathrm{MeV}$. Total M1 strength of 4.17(50) $\mu_{\mathrm{N}}^{2}$ with a centroid of $9 \mathrm{MeV}$ was found in a photon-scattering experiment with monoenergetic and $100 \%$ linearly polarized beams. More than $40 \mathrm{~J}^{+} 1^{+}$states have been identified from observed ground-state transitions [17].

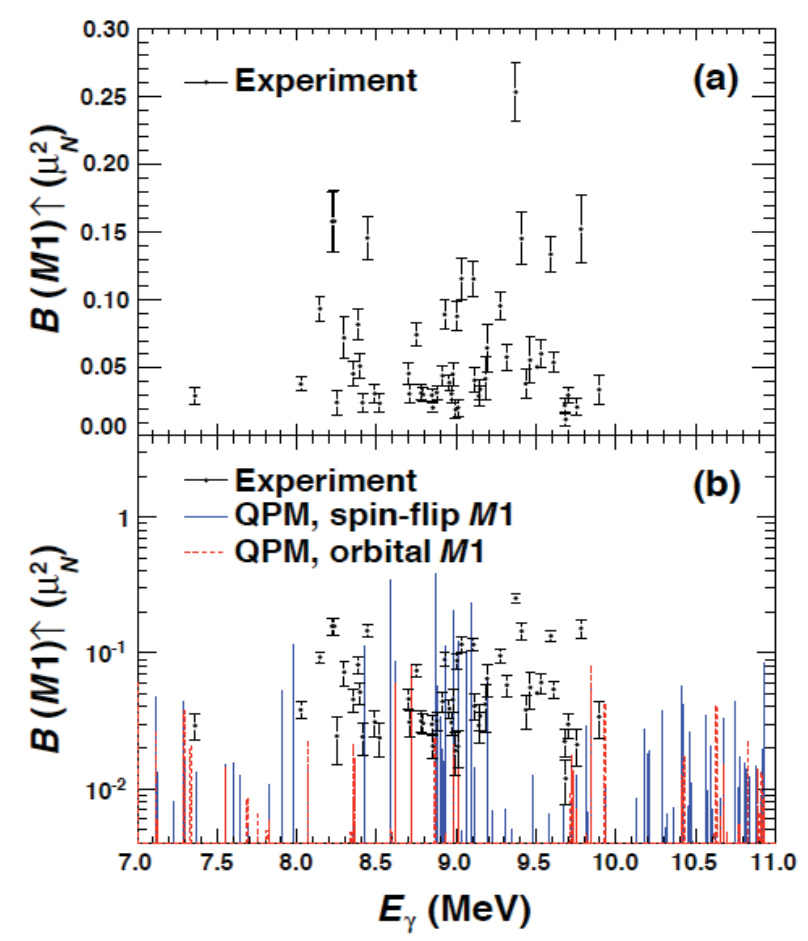

Figure 2. Results for (a) the measured M1 strength of discrete levels in ${ }^{90} \mathrm{Zr}$ and (b) predictions from the quasiparticle-phonon model [17]. 
The deduced M1 values in ${ }^{90} \mathrm{Zr}$ are shown in Fig.2. The experiment shows a rather fragmented excitation spectrum with many $1^{+}$states, each of them with a strength below $1 \mu_{\mathrm{N}}{ }^{2}$. The fragmentation pattern of the M1 strength below and above the neutron-separation energy $\left(\mathrm{S}_{\mathrm{n}}=12.0 \mathrm{MeV}\right)$ was obtained with an extended version of the QPM [18]. M1 transitions are calculated with a quenched effective spin-magnetic factor $g_{\text {eff }}^{s}=0.8$ $g_{\text {bare }}{ }^{s}$. The analysis of the QRPA M1 strength of $1^{+}$-state excitations with energies up to $20 \mathrm{MeV}$ indicates that it is mostly due to single $p$ - $h$ spin-flip states. One surprising result from these calculations is that the M1 strength at excitation energies between 7 and $11 \mathrm{MeV}$ contains a considerable orbital part of about $22 \%$. Nevertheless, the total orbital QRPA strength for the whole energy range up to $20 \mathrm{MeV}$ is very small, less than $2 \%$ of the total QRPA M1 strength. An interference between spin and orbital strengths leads to the suppression of the total M1 response.

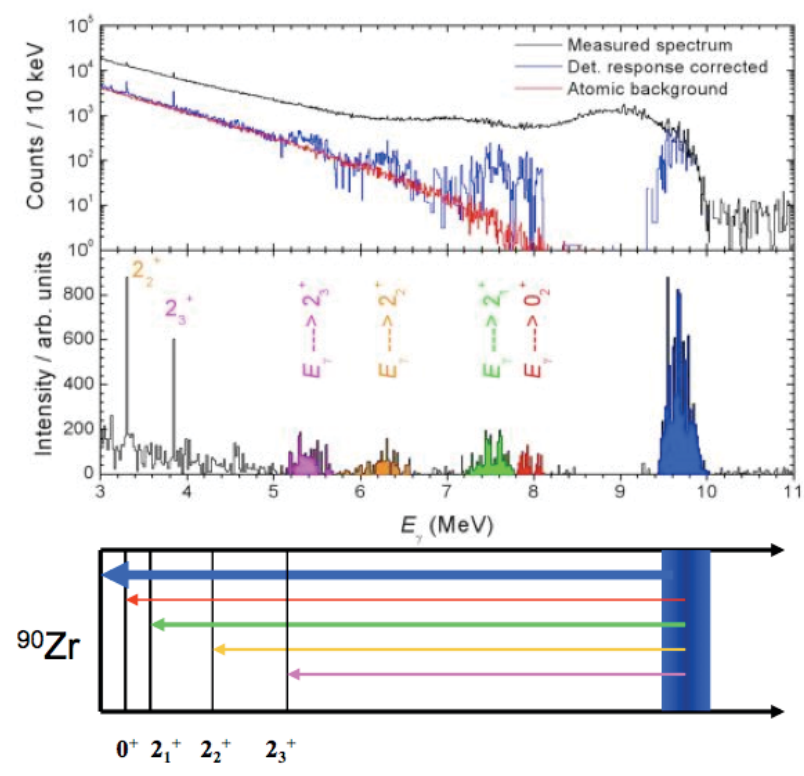

Figure 3. The unfolding algorithm applied to HPGe spectrum measured with ${ }^{90} \mathrm{Zr}$ target at $9.8 \mathrm{MeV}$ photon energy. The lower part shows the primary transitions to the first excites states.

An additional observation was exploited in ${ }^{90} \mathrm{Zr}$ measurements to investigate the nature of the PDR and collect complementary information on the decay pattern of the PDR states. This includes studying the branching ratios of the PDR states to the low-lying excited states. The partial decay width $\Gamma_{i}$ of transitions to low-lying excited states or to the ground state are directly linked to the electromagnetic transition matrix elements between the corresponding states. Therefore, each decay channel is sensitive to a different component in the wave function. The observation of these transitions and the determination of branching ratios revealed important experimental information, which is needed to provide stringent and sensitive tests to modern model calculations. Extracting the underlying spectrum from a measured spectrum with a known detector response function is shown on Fig. 3. The measured spectrum with incident photons of $9.8 \mathrm{MeV}$ and energy spread of 260
$\mathrm{keV}$ is shown on the top panel (black histogram). Atomic background stemming from non-resonant scattering is shown by the red curve. The resulting unfolded spectrum, after subtraction of the atomic background, with the intensity distribution of the primary $\gamma$-ray transitions is shown in the panel below. The primary transitions decaying to the first four excited states in ${ }^{90} \mathrm{Zr}$ are shown in the level scheme below the unfolded spectrum. As can be seen, the unfolding method [29] revealed important decay information about the intensity and the distribution of the primary $\gamma$-ray transitions stemming from the PDR.

\subsection{Magnetic dipole strength in ${ }^{206} \mathrm{~Pb}$}

The strength and the location of the M1 resonance in medium-and heavy-mass nuclei has been a long-standing problem in nuclear structure physics because of the large discrepancies between experimental and theoretical results [31]. Prediction of the magnitude and the distribution of the M1 strength is a major problem for theory since there is no model-independent sum rule. In general, the total M1 strength depends on the properties of the ground-state wave function. The experimental deficiency of the observed M1 strength in comparison to the predicted one was called quenching.

Most of the experimental and theoretical works of the M1 spin-flip resonance were devoted to the doubly magic ${ }^{208} \mathrm{~Pb}$ nucleus $[3,21] .{ }^{206} \mathrm{~Pb}$ has two neutrons less than ${ }^{208} \mathrm{~Pb}$, but the excitation spectra of these two nuclei differ significantly. Passing from the magic ${ }^{208} \mathrm{~Pb}$ nucleus to its neighbor results in much higher density of complex configurations in ${ }^{206} \mathrm{~Pb}$ at the same excitation energies. Hence, there is an expectation of strong fragmentation of the M1 strength in ${ }^{206} \mathrm{~Pb}$ in comparison to ${ }^{208} \mathrm{~Pb}$. The neutron separation energy of ${ }^{208} \mathrm{~Pb}$ is $7.4 \mathrm{MeV}$ and very close to where the isovector M1 resonance should be located [20] while the neutron separation energy of ${ }^{206} \mathrm{~Pb}$ is $8.1 \mathrm{MeV}$. Hence, a larger part of the total M1 strength in ${ }^{206} \mathrm{~Pb}$ can be observed with real photons.

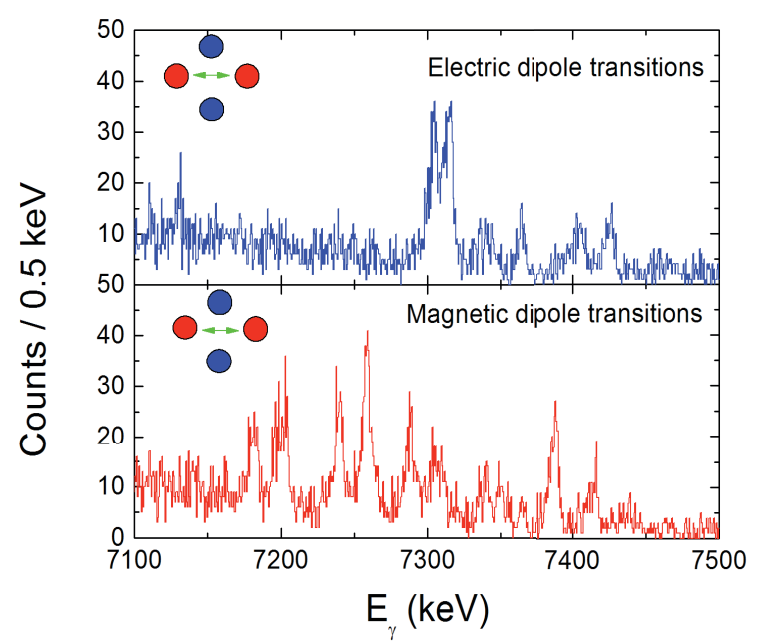

Figure 4. Photon-scattering spectra observed at $\theta= \pm 90^{\circ}$ in the plane parallel (bottom panel) and perpendicular to the polarization plane (top panel) of the HIGS photon beam at $E_{v}=$ 7.3 MeV. The energy spread (FWHM) is about $220 \mathrm{keV}$. 
We measured the spin-flip $M 1$ strengths in ${ }^{206} \mathrm{~Pb}$ have been measured in a photon-scattering experiment. The HIGS beams in combination with high-resolution HPGe detectors, positioned at specific angles relative to the initially $100 \%$ linearly polarized photon beams, open up the possibility to precisely deducing the strengths and locations of individual M1 transitions, comprising the giant M1 resonance, and to distinguish them unambiguously from the E1 and E2 deexcitations. Two $\gamma-$ ray spectra from vertical (top panel) and horizontal (bottom panel) $60 \%$ HPGe detectors at a beam energy of 7.3 MeV is shown are Fig. 4.

First preliminary results of the electric- (top panel) and magnetic-dipole (bottom panel) strength in ${ }^{206} \mathrm{~Pb}$ are shown in Fig. 5. As can be seen the magnetic M1 strength is concentrated in two energy regions around 6.0 and 7.3 $\mathrm{MeV}$. It should be noted that this strength was obtained from the elastic dipole (E1 or M1) transitions in ${ }^{206} \mathrm{~Pb}$. A considerable amount of inelastic strength stemming from high excitation energies, near the neutron-separation energy, is observed from the first excited states.

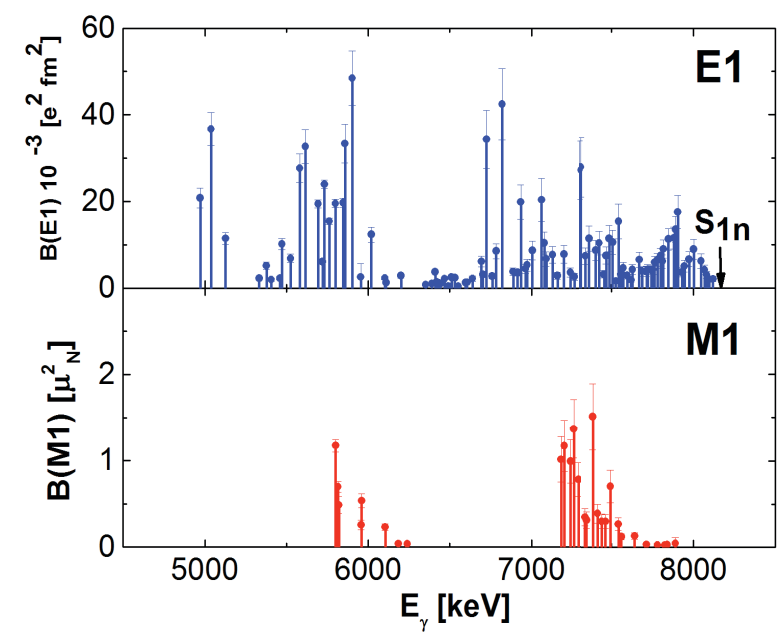

Figure 5. Electric (upper panel) and magnetic (lower panel) dipole strength distribution in ${ }^{206} \mathrm{~Pb}$.

\section{Indirect neutron capture cross-section measurement on unstable ${ }^{85} \mathrm{Kr}$ nucleus}

The amount of ${ }^{86} \mathrm{Kr}$ produced by the $s$-process is determined by the branching point at the unstable ${ }^{85} \mathrm{Kr}$, which has a half-life of 10.76 years. When the $s$-process reaches this isotope, two events can occur. If the neutron density remains fairly low, it will decay to ${ }^{85} \mathrm{Rb}$ as shown in Fig. 6. However, if the neutron density is high $\left(\geq 5 \times 10^{8}\right.$ $\left.\mathrm{n} / \mathrm{cm}^{3}\right)$, more than $50 \%$ of the time ${ }^{85} \mathrm{Kr}$ will capture a neutron and make ${ }^{86} \mathrm{Kr}$. The measured isotopic composition of $\mathrm{Kr}$ clearly reveals the signature of the $s$ (slow neutron-capture) process. It is thought that $\mathrm{Kr}$ is ionised and implanted in stardust $\mathrm{SiC}$ grains via stellar winds in two different energy components: one during the AGB phase in small grains showing low ${ }^{86} \mathrm{Kr} /{ }^{82} \mathrm{Kr}$, and another during the post-AGB phase in large grains showing high ${ }^{86} \mathrm{Kr} /{ }^{82} \mathrm{Kr}$ ratios [27].

There are a few reasons why the capture reaction on ${ }^{85} \mathrm{Kr}$ is important. Firstly, the branching at ${ }^{85} \mathrm{Kr}$ is significant for s-process modeling. Secondly, this branching is independent of the temperature and depends only on the neutron density. Thirdly, it helps to constrain the neutron density parameter in models of AGB stars. And finally, the ${ }^{85} \mathrm{Kr}$ neutron capture has not been measured.

The present measurements were carried out at HIGS facility. The target consisted of $1012 \mathrm{mg}$ of $\mathrm{Kr}$ gas enriched to $99.4 \%$ in ${ }^{86} \mathrm{Kr}$, contained in a stainless steel cell. An empty cell of identical material and dimension was used to subtract the background contribution. The neutrons emitted from the ${ }^{86} \operatorname{Kr}(\gamma, n)$ reaction were detected using a $4 \pi$ assembly of ${ }^{3} \mathrm{He}$ proportional counters.

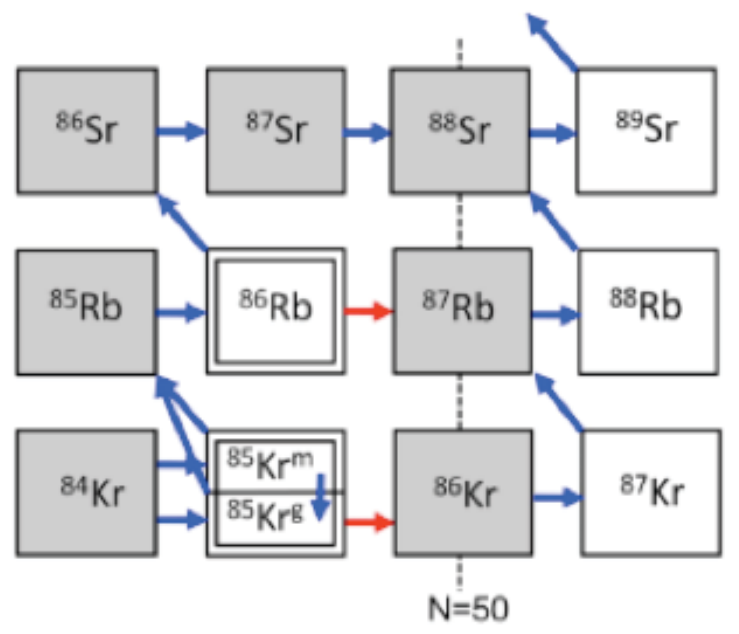

Figure 6. Isotopic map near the $s$-process branching at ${ }^{85} \mathrm{Kr}$. Isotopes shown as shaded (white) squares are stable (radioactive). The blue (red) arrows depict the $s$-process path at low (high) neutron densities. The ground state of ${ }^{85} \mathrm{Kr}$ can either $\beta$ decay $\left(\mathrm{T}_{1 / 2}=10.75 \mathrm{y}\right)$ to ${ }^{85} \mathrm{Rb}$ or capture a neutron to form ${ }^{86} \mathrm{Kr}$. The isomeric state of ${ }^{85} \mathrm{Kr}$ can either $\beta$ decay $\left(\mathrm{T}_{1 / 2}=4.48\right.$ h) or decay to the ground state via $\gamma$-ray emission.

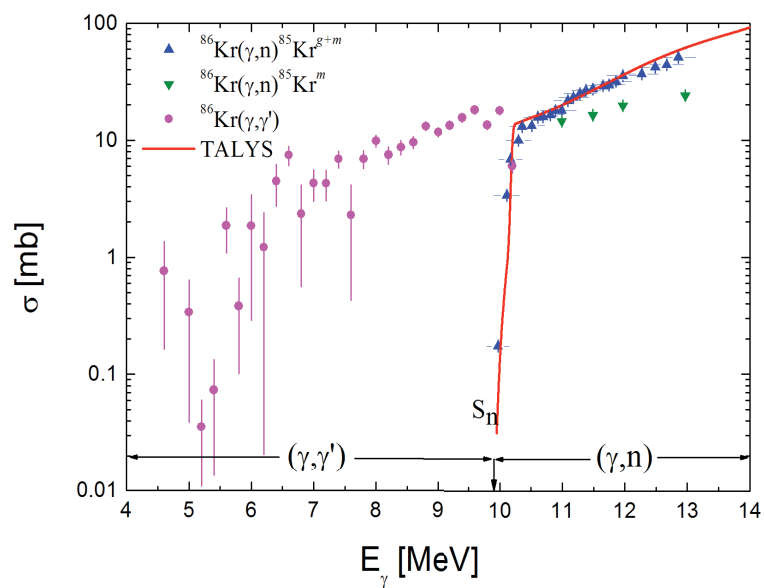

Figure 7. The blue point-up triangles represent the total cross section of the ${ }^{86} \mathrm{Kr}(\gamma, \mathrm{n})^{85} \mathrm{Kr}^{\text {tot }}$ reaction while the green pointdown triangles denote the ${ }^{86} \mathrm{Kr}(\gamma, \mathrm{n})^{85} \mathrm{Kr}^{\mathrm{m}}$ cross section, both from the present measurements. The purple circles show the data from the ${ }^{86} \mathrm{Kr}\left(\gamma, \gamma^{\prime}\right)^{86} \mathrm{Kr}$ reaction [22]. The calculated cross section for the ${ }^{86} \mathrm{Kr}(\gamma, \mathrm{n}){ }^{85} \mathrm{Kr}^{\text {tot }}$ reaction, using the TALYS code, is represented by the red line. 
The cross-section values from the present measurements are plotted in Fig. 7. As shown, the $\gamma$-strength function is now fully constrained at all photon energies relevant to the $s$ process by our experimental data, below and above the neutron-separation energy [24]. The experimental $\gamma$ ray strength function, obtained by both the $(\gamma, n)[23]$ and the $\left(\gamma, \gamma^{\prime}\right)$ measurements [22], was directly included in the TALYS code [28], in a tabulated and interpolated form, for calculating the neutron capture cross section of ${ }^{85} \mathrm{Kr}$. The transitional region around the neutron threshold has been described by the QRPA model, to reproduce the experimental data fairly well. The result of this calculation is presented in Fig. 8. The estimated uncertainties (shaded area) are dominated by the experimental errors of the $\gamma$-ray strength function (see Fig. 7) and the assumption for the nuclear level density.

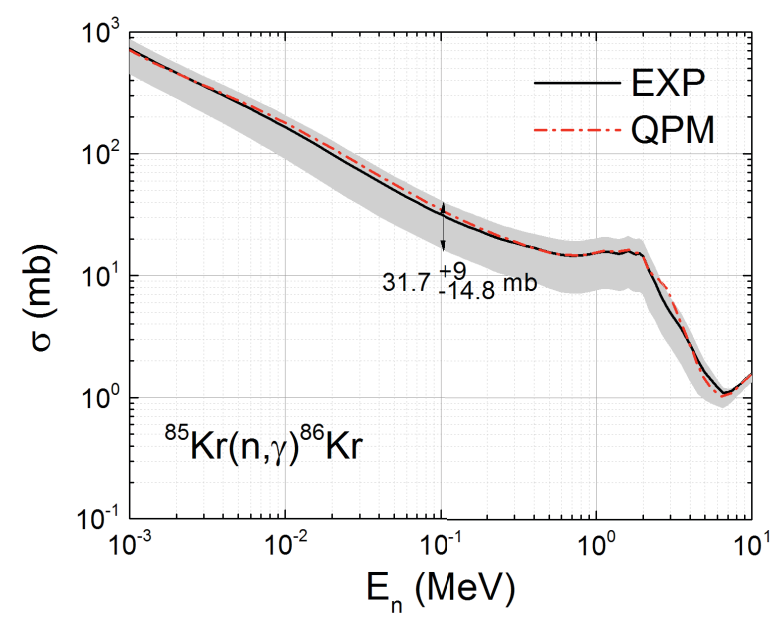

Figure 8. Cross section of ${ }^{85} \mathrm{Kr}(\mathrm{n}, \gamma)^{86} \mathrm{Kr}$ calculated with TALYS using experimental dipole (in black) and three-phonon quasiparticle-phonon model (QPM) strength functions (in red). The predicted uncertainties (shaded area) are derived from the experimental errors of the dipole strength function and from variations in the nuclear level density parameters.

At a stellar temperature of $\mathrm{kT}=30 \mathrm{keV}$, our new Maxwellian-averaged cross section amounts to a value of $83 \pm 23 \mathrm{mb}$. This value is about $50 \%$ higher than the result of Ref. [30] quoted above. Furthermore, the uncertainty is improved by a factor of about 3 to $\approx 50 \%$.

\section{Conclusions}

Systematic spin and parity measurements on spherical and near spherical nuclei at the HIGS facility have verified that the observed dipole strength from $4 \mathrm{MeV}$ to the neutron-separation energy is predominantly of electric nature. Our findings are in agreement with the QPM predictions for the character and strength of this dipole excitation mode. The deduced photoabsorption cross section exhibits a resonance-like shape distribution with pronounced peak structure. In addition, the fine structure of the M1 "spin-flip" mode was observed in ${ }^{90} \mathrm{Zr}$ and ${ }^{206} \mathrm{~Pb}$ nuclei.

\section{Acknowledgments}

This work was supported by the DOE grants DE-FG0297ER41033, DE-FG02-97ER41033, DE-FG02 97ER41042, DE-FG02-97ER41041, DFG grant Le439/6 and performed under the auspices of the Lawrence Livermore National Security, LLC, (LLNS) under Contract No. DEAC52-07NA27344.

\section{References}

1. G. A. Bartholomew et al., Adv. Nucl. Phys. 7, 229 (1972)

2. A. Zilges et al., Phys. Lett. B 542, 43 (2002)

3. N. Ryezayeva et al., Phys. Rev. Lett. 89, 272502 (2002)

4. S. Volz et al., Nucl. Phys. A 779, 1 (2006)

5. J. Enders et al., Nucl. Phys. A 741, 3 (2004)

6. D. Savran et al., Phys. Rev. Lett. 100, 232501 (2008)

7. G. Rusev et al., Phys. Rev. C 73, 044308 (2006)

8. D. Savran et al., Phys. Rev. Lett. 97, 172502 (2006)

9. R. Schwengner et al., 78, 064314 (2008)

10. A.P. Tonchev et al, CGS14 proceeding, Guelph, Canada, 2011

11. T. Inakura et al., Phys. Rev. C, 84, 021302(R) (2011)

12. P.M. Goddard et al., Phys. Rev. C 88, 064308 (2013)

13. C. Romig et al., Phys. Rev. C 88, 044331 (2013)

14. M. Scheck et al., Phys. Rev. C 87, 051304(R) (2013)

15. R. Massarczyk et al., Phys. Rev. Lett. 112, 072501 (2014)

16. V. Derya et al., Phys. Lett. B. 730, 288 (2014)

17. G. Rusev et al., Phys. Rev. Lett. (2013)

18. N. Tsoneva et al., Phys. Rev. C 77, 024321 (2008)

19. A.P. Tonchev et al., Phys. Rev. Lett. 104, 072501 (2010)

20. V. Ponomarev et al., J. Phys. G: Nucl. Phys., 13, 1523 (1987)

21. T. Shizuma et al., Phys. Rev. C 78, 061303(R) (2008)

22. R. Schwengner at al., Phys. Rev. C. 87, 024306 (2013)

23. R. Raut et al., Phys. Rev. Lett. 111, 112501 (2013)

24. A. Sauerwein et al., Phys. Rev. C 89, 035803 (2014)

25. R. Mohan, M. Danos, L. Biedenharn, Phys. Rev. C 3, 1740 (1971)

26. S. Goriely and E. Khan, Nucl. Phys. A 706, 217 (2002)

27. M. Lugaro, Stardust from Meteorites (World Scientific, Singapore, 2005)

28. S. Goriely, S. Hilaire, and A.J. Koning, Astron. Astrophys. 487, 767 (2008)

29. G. Rusev. PhD dissertation FZD-478 (2007)

30. Z. Y. Bao et al, At. Data Nucl. Data Tables 76, 70 (2000)

31. K. Heyde et al. Rev. Mod., Phys. 82, 2365 (2010)

32. D. Savran et al., Prog. Part. Nucl. Phys. 70, 210 (2013)

33. S.P. Kamerdzhiev and S.F. Kovalev. Phys. Atom. Nucl. 69, 418 (2006) 
